EPJ Web of Conferences 69, 00009 (2014)

DOI: $10.1051 /$ epjconf / 20146900009

(C) Owned by the authors, published by EDP Sciences, 2014

\title{
Few-body semiclassical approach to nucleon transfer and emis- sion reactions
}

\author{
Renat A. Sultanov ${ }^{1, a}$ and D. Guster ${ }^{1, b}$ \\ ${ }^{1}$ Department of Information Systems \& Integrated Science and Engineering Laboratory Facilities (ISELF), \\ St. Cloud State University, St. Cloud, MN 56301-4498, USA
}

\begin{abstract}
A three-body semiclassical model is proposed to describe the nucleon transfer and emission reactions in a heavy-ion collision. In this model the two heavy particles, i.e. nuclear cores $\mathrm{A}_{1}\left(Z_{A_{1}}, M_{A_{1}}\right)$ and $\mathrm{A}_{2}\left(Z_{A_{2}}, M_{A_{2}}\right)$, move along classical trajectories $\vec{R}_{1}(t)$ and $\vec{R}_{2}(t)$ respectively, while the dynamics of the lighter neutron (n) is considered from a quantum mechanical point of view. Here, $M_{i}$ are the nucleon masses and $Z_{i}$ are the Coulomb charges of the heavy nuclei $(i=1,2)$. A Faddeev-type semiclassical formulation using realistic paired nuclear-nuclear potentials is applied so that all three channels (elastic, rearrangement and break-up) are described in a unified manner. In order to solve the time-dependent equations the Faddeev components of the total three-body wave-function are expanded in terms of the input and output channel target eigenfunctions. In the special case, when the nuclear cores are identical $\left(A_{1} \equiv A_{2}\right)$ and also the two-level approximation in the expansion over the target (subsystem) functions is used, the time-dependent semiclassical Faddeev equations are resolved in an explicit way. To determine the realistic $\vec{R}_{1}(t)$ and $\vec{R}_{2}(t)$ trajectories of the nuclear cores, a self-consistent approach based on the Feynman path integral theory is applied.
\end{abstract}

\section{Introduction}

When trying to describe nuclear collisions, compound and halo nuclei, or, for instance, complex nuclear fusion reactions, few-body models are extremely useful and can play a very important role in the field [1,2]. For example, in works [3-5] the authors widely use various few-body models of complex nuclei for numerical computation of different systems and nuclear reactions. In an older paper [6] a detailed few-body approach has been developed for calculation of an important problem in nuclear astrophysics, namely the first two $0^{+}$levels in the nucleus ${ }^{12} \mathrm{C}$ which was considered as a model three $\alpha$-particle system [7-9]. Specifically, well known three-body Faddeev equations [10] were used in $[6,9]$. Further, in the case of heavy-ion collisions a three-body semiclassical model has been introduced in $[11,12]$. Once again a Faddeev-type formulation was utilized featuring singleterm separable (non-local) potentials between particles. For solution of the few-body equations a simplified semiclassical model was applied, where heavier nuclear cores of the system followed along straight-line classical trajectories. Therefore, the resulting model featuring the semiclassical Faddeev

\footnotetext{
a e-mail: rasultanov@stcloudstate.edu

be-mail: dcguster@stcloudstate.edu
}

This is an Open Access article distributed under the terms of the Creative Commons Attribution License 2.0, which permits unrestricted use, distribution, and reproduction in any medium, provided the original work is properly cited. 


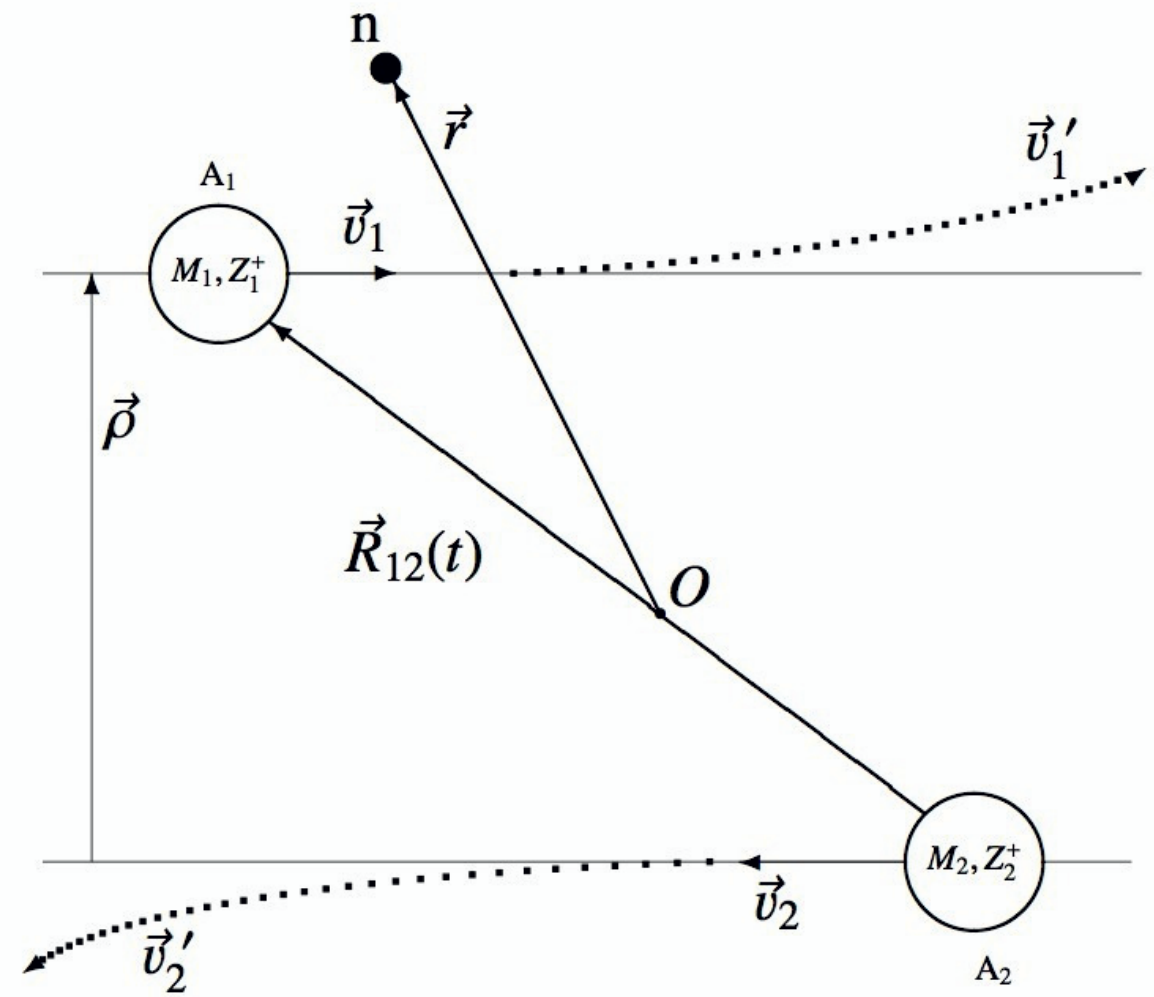

Figure 1. Neutron (n) few-body quantum dynamics between two classically moving nuclear cores: $\mathrm{A}_{1}=\left(M_{1}, Z_{1}^{+}\right)$ and $\mathrm{A}_{1}=\left(M_{2}, Z_{2}^{+}\right)$. Here, $M_{i}, Z_{i}^{+}$are the masses and Coulomb charges respectively of $\mathrm{A}_{i}(i=1,2), O$ is the centerof-mass of the three body system, $\vec{r}$ is the coordinate radius-vector of $\mathrm{n}, \vec{R}_{12}(t)=\vec{R}_{1}(t)-\vec{R}_{2}(t)$ is the separation vector between $\left(M_{1}, Z_{1}^{+}\right)$and $\left(M_{2}, Z_{2}^{+}\right), \vec{R}_{1}(t)$ and $\vec{R}_{2}(t)$ are the radius-vectors of $\mathrm{A}_{1}$ and $\mathrm{A}_{2}\left(\vec{R}_{1(2)}(t)\right.$ are not shown in this figure), $t$ is the time in the system, $\vec{v}_{1}$ and $\vec{v}_{2}$ are initial velocities of $\left(M_{1}, Z_{1}^{+}\right)$and $\left(M_{2}, Z_{2}^{+}\right)$at $t \rightarrow-\infty$, respectively, $\vec{v}_{1}^{\prime}$ and $\vec{v}_{2}^{\prime}$ are final at $t \rightarrow+\infty$ velocities of $\left(M_{1}, Z_{1}^{+}\right)$and $\left(M_{2}, Z_{2}^{+}\right)$respectively, $\rho$ is the impact parameter of the three-body collision: $\left[\left(M_{1}, Z_{1}^{+}\right), \mathrm{n}\right]+\left(M_{2}, Z_{2}^{+}\right) \rightarrow\left[\left(M_{2}, Z_{2}^{+}\right), \mathrm{n}\right]+\left(M_{1}, Z_{1}^{+}\right) ;\left(M_{2}, Z_{2}^{+}\right)+\left(M_{1}, Z_{1}^{+}\right)+\mathrm{n}$, where the nucleon transfer from $\left(M_{1}, Z_{1}^{+}\right)$to $\left(M_{2}, Z_{2}^{+}\right)$and the three-body break-up channels are presented here. The nuclear interaction between $\mathrm{n}$ and $\mathrm{A}_{i}(i=1,2)$ depends on the distances $|\vec{x}|$ and $|\vec{y}|$ between $\mathrm{n}$ and $\mathrm{A}_{1}$ and between $n$ and $\mathrm{A}_{2}$ respectively ( $\vec{x}$ and $\vec{y}$ are not shown in this figure).

equations became a set of coupled time-dependent integral equations. More generally, in the case of heavy ion collisions [13], various semiclassical models have been formulated and applied, see for example [14-16]. However, these approaches mainly used simple straight-line model trajectories [17]. Also, there are other interesting and important problems in the field of heavy-ion collisions such as neutron and charge transfer and emission reactions [18]. In the framework of the Faddeev approach these channels can be treated in a unified manner.

Nontheless, in Ref. [15] an interesting attempt has been made to expand this process. In this work the author tried to apply a semiclassical Pechukas formalism [19] to obtain more realistic clas- 
sical trajectories of heavy nuclear particles. Pechukas's method was originally developed for atomic, molecular collisions and chemical reactions. This theory expands on Feynman's interpretation of quantum-mechanics, which is based on path integrals [20]. Usually, semiclassical methods and models, such as [19], allow us to gain even deeper insight into different few-body or many-body physical systems. They also enable us to introduce even more realistic classical trajectories of heavier particles in models, i.e. to take into account quantum-mechanical corrections in a self-consistent manner [19].

Generally speaking, such a combination of few-body models and methods together with semiclassical models, where the dynamics of heavier particles can be separated from the dynamics of lighter particles, seems to be quite useful. The same approach has already been developed and widely used in several problems of chemical physics for molecular dynamics [21] and even for the description of many body systems, see for example [22].

In the current work we develop a semiclassical model for a few-body treatment of neutron transfer and emission reactions in heavy ion collisions at different impact energies. The $\left(\mathrm{A}_{1}, n\right)+\mathrm{A}_{2}$ system is shown in Fig. 1, where $\mathrm{A}_{1}$ and $\mathrm{A}_{2}$ are the heavy nuclear cores which move along classical trajectories $\vec{R}_{1}(t)$ and $\vec{R}_{2}(t)$. The inter-distance vector $\vec{R}_{12}(t)=\vec{R}_{1}(t)-\vec{R}_{2}(2)$ is also shown in Fig. 1 together with the coordinate $\vec{r}$ of the third particle, i.e. neutron n, $\vec{\rho}$ is the impact parameter, $\vec{v}_{1}\left(\vec{v}_{1}{ }^{\prime}\right)$ and $\vec{v}_{2}\left(\vec{v}_{2}{ }^{\prime}\right)$ are the initial (final) velocities of the heavy particles, $O$ is the center-of-mass of the three-body system. The semiclassical model of a time-dependent set of Faddeev equations is used. However, in contrast to Revai's approach $[11,12]$ we formulate the model with the use of two local (realistic) paired nuclearnuclear potentials between the $\mathrm{A}_{1}$ particle and $\mathrm{n}$ and between $\mathrm{A}_{2}$ and $\mathrm{n}$. The heavy nuclei $\mathrm{A}_{1}\left(Z_{A_{1}}, M_{A_{1}}\right)$ and $\mathrm{A}_{2}\left(Z_{A_{2}}, M_{A_{2}}\right)$ move along realistic classical trajectories $\vec{R}_{1}(t)$ and $\vec{R}_{2}(t)$, while the motion of the relatively light neutron $\mathrm{n}\left(m_{n} \ll M_{A_{i}}\right)$ in their nuclear fields is treated from a quantum-mechanical point of view. In this model the heavy particles can move along complex Coulomb trajectories. This problem is particularly important for lower energy collisions and small impact parameters, i.e. at $\rho \approx 0$ : when the use of simple straight-line trajectories does not provide an appropriate approximation [23]. In this work we employ a self-consistent Pechukas method [19] which provides a proper way to determine the true trajectories of the heavy classical particles [15, 23]. In the next section we will delineate our semiclassical formalism. The self-consistent Pechukas approach is also explained and applied to the three-body semiclassical system as shown in Fig. 1.

\section{Semiclassical model}

In this section a few-body semiclassical model for a single neutron (n, particle 3), transition from one heavy center to another (particles 1 and 2) and n-emission process when the particle reaches the continuous spectrum is presented. Specifically, we consider the following three channel reaction:

$$
\begin{aligned}
(13)+2 & \rightarrow(13)+2 \\
& \rightarrow(23)+1 \\
& \rightarrow 1+2+3 .
\end{aligned}
$$

In order to describe these processes in a unified way the few-body Faddeev equation approach is applied. To solve these equations a modified close coupling method [24, 25] is used. This method provides a set of coupled time-dependent differential equations with unknown expansion coefficients.

\subsection{Time-dependent few-body Faddeev equations}

The time-dependent integral differential Faddeev equations [10] can be delineated:

$$
\left(i \hbar \frac{\partial}{\partial t}-\hat{H}_{0}-V_{j k}\right)\left|\Psi_{l}\right\rangle=V_{j k}\left(\left|\Psi_{j}\right\rangle+\left|\Psi_{k}\right\rangle\right),
$$


where $H_{0}$ is the kinetic energy operator of the three particles:

$$
\hat{H}_{0}=-\frac{\hbar^{2}}{2 \mu_{j k}} \Delta_{\vec{r}_{j k}}+\frac{\hbar^{2}}{2 M_{l}} \Delta_{\vec{R}_{l}}=\hat{T}_{\vec{r}_{j k}}+\hat{T}_{\vec{R}_{l}}
$$

$\vec{r}_{j k}$ and $\vec{R}_{l}$ are the Jacobi coordinates, $\mu_{j k}$ and $M_{l}$ the corresponding reduced masses, $V_{j k}$ the two-body potentials, $\left|\Psi_{l}\right\rangle$ are the Faddeev components, and $j \neq k \neq l=1,2,3$. As mentioned above we consider the third particle (neutron or even $\alpha$-particle) to be the light one, i.e.:

$$
\frac{m_{n_{0}}}{M_{A_{1}}} \ll 1, \quad \frac{m_{n_{0}}}{M_{A_{2}}} \ll 1,
$$

where $m_{n_{0}}$ is the neutron mass, and $M_{A_{1}}$ and $M_{A_{2}}$ are masses of the heavy nuclei. Then, the heavy particles 1 and 2 can be considered as moving along classical trajectories $\vec{R}_{1}(t)$ and $\vec{R}_{2}(t)$, while the motion of lighter particles (n or, say $\alpha$-particle) can be described quantum-mechanically. The situation is shown in Fig. 1. Putting $M_{A_{1}} \sim M_{A_{2}} \sim \infty$, we come to $\hat{T}_{\vec{R}_{1}} \sim \hat{T}_{\vec{R}_{2}} \sim 0$ and one can obtain a set of semiclassical equations. Therefore, for the treatment of this situation and description of the light particle dynamics we use instead of three coupled, time-dependent Faddeev equations (2) just two Faddeev-like equations [26-28]:

$$
\begin{aligned}
& \left(i \hbar \frac{\partial}{\partial t}-\frac{\hat{p}_{r}^{2}}{2 m_{n_{0}}}-V_{13}(\vec{x})-\hat{\xi}_{1}\right) \Psi_{1}(\vec{r}, \vec{R}(t), t)=\left(V_{13}(\vec{x})-\hat{\xi}_{2}\right) \Psi_{2}(\vec{r}, \vec{R}(t), t), \\
& \left(i \hbar \frac{\partial}{\partial t}-\frac{\hat{p}_{r}^{2}}{2 m_{n_{0}}}-V_{23}(\vec{y})-\hat{\xi}_{2}\right) \Psi_{2}(\vec{r}, \vec{R}(t), t)=\left(V_{23}(\vec{y})-\hat{\xi}_{1}\right) \Psi_{1}(\vec{r}, \vec{R}(t), t) .
\end{aligned}
$$

Here, $\vec{R}(t)$ is the relative vector between particles $\mathrm{A}_{1}$ and $\mathrm{A}_{2}$, where the time dependence is determined according to classical mechanics. The dynamics of the light particle 3 is treated quantum mechanically: $\vec{p}_{r}=\hbar \vec{\nabla}_{r} / i$ is the momentum operator corresponding to the relative variable $\vec{r}$ between the third particle $n$ and the center of mass of particles $\mathrm{A}_{1}$ and $\mathrm{A}_{2}$. The relative vectors in the subsystems (13) and (23) are denoted by $\vec{x}$ and $\vec{y}$, respectively, and arbitrary operators $\hat{\xi}_{1}$ and $\hat{\xi}_{2}$ may allow us to construct few-body coupled equations with specific preset features.

In the semiclassical approach the total Hamiltonian of the three-body system may be written in the following form:

$$
\hat{h}=\frac{\hat{p}_{r}^{2}}{2 m_{n_{0}}}+V_{23}(|\vec{x}|)+V_{13}(|\vec{y}|)+U\left(\vec{R}_{12}\right)=\hat{H}(t)+U\left(\vec{R}_{12}\right),
$$

where $\vec{x}$ and $\vec{y}$ are the distances between the light particle and the two heavier classically moving particles, $\vec{R}_{12}$ is the distance between them. Actually, $U\left(R_{12}^{t}\right)=V_{12}$ is a constant or c-number in the $\vec{r}$ - Hilbert space. Therefore, in Eqs. (5)-(6) we obtain:

$$
V_{12}=0, \quad\left|\Psi_{3}\right\rangle=0 .
$$

Hence, the total wave function of the three-particle system (123), see Fig. 1, can be presented by only two relevant components:

$$
|\Psi\rangle=\left|\Psi_{1}\right\rangle+\left|\Psi_{2}\right\rangle .
$$

As we already mentioned above the additional operators $\hat{\xi}_{1}$ and $\hat{\xi}_{2}$ introduced in (5)-(6) can help to obtain few-body equations with preset properties. For example, if we assume that

$$
\hat{\xi}_{1}=\frac{\hat{p}_{r}^{2}}{2 M_{A_{1}}}
$$


and

$$
\hat{\xi}_{2}=\frac{\hat{p}_{r}^{2}}{2 M_{A_{2}}},
$$

then, in the left hand sides of the time-dependent Faddeev-like equations (5)-(6) we obtain proper Hamiltonians of the (23) and (13) subsystems, i.e. instead of

$$
\hat{h}_{k 3}=\frac{\hat{p}_{r}^{2}}{2 m_{n_{0}}}+V_{k 3}\left(\left|\vec{r}-\vec{R}_{k}(t)\right|\right),
$$

one can obtain the more realistic and asymptotically correct two-body Hamiltonian, i.e.

$$
{\hat{h^{\prime}}}_{k 3}=\frac{\hat{p}_{r}^{2}}{2 m_{n}}+V_{k 3}\left(\left|\vec{r}-\vec{R}_{k}(t)\right|\right) .
$$

Here, $m_{n}(k)=m_{n_{0}} M_{A_{k}} /\left(m_{n_{0}}+M_{A_{k}}\right)$ are the reduced masses of the targets, $k=1,2$.

To solve these equations (5)-(6), we expand the wave function components $\Psi_{k}(\vec{r}, \vec{R}(t), t)$ into the solutions $\Phi_{n}^{(k 3)}(\vec{r}, \vec{R}(t), t)$ of the respective subsystem's Schrödinger equation:

$$
\left(i \hbar \frac{\partial}{\partial t}-\frac{\hat{p}_{r}^{2}}{2 m_{n}}-V_{k 3}\left(\vec{r}-\vec{R}_{k}(t)\right)\right) \Phi_{n}^{(k 3)}\left(\vec{r}, \vec{R}_{k}(t), t\right)=0 .
$$

That is, we can write

$$
\Psi_{k}(\vec{r}, \vec{R}(t), t)=\left(\sum+\int\right)_{n} C_{n}^{(k)}(\vec{R}(t), t) \Phi_{n}^{(k 3)}(\vec{r}, \vec{R}(t), t),
$$

in which the summation (integration) runs across the whole discrete and continuous spectrum. For a constant velocity $\dot{\vec{R}}_{k}(t)=\vec{v}_{k}$ one finds [26-28]

$$
\Phi_{n}^{(k 3)}(\vec{r}, \vec{R}(t), t)=e^{i m_{n} \vec{v}_{k} \vec{r} / \hbar-i\left(E_{n}^{k 3}+\frac{m_{n}}{2} v_{k}^{2}\right) t / \hbar} \varphi_{n}^{(k 3)}\left(\vec{r}-\vec{R}_{k}(t)\right),
$$

the functions $\varphi_{n}^{k 3}$ being given by

$$
\left(-\frac{\hbar^{2}}{2 m_{n}} \Delta_{\vec{x}}+V_{k 3}(\vec{x})\right) \varphi_{n}^{(k 3)}(\vec{x})=E_{n}^{(k 3)} \varphi_{n}^{(k 3)}(\vec{x}) .
$$

Inserting the expansion (15) into (5)-(6), we obtain for the coefficients $C_{n}^{(k)}$ a set of coupled equations:

$$
\begin{aligned}
& i \hbar \frac{\partial C_{n}^{(1)}(\vec{R}(t), t)}{\partial t}=\left(\sum+\int\right)_{m} W_{n m}^{12}(R(t), t) \gamma_{n m}^{(12)}(t) C_{m}^{(2)}(\vec{R}(t), t), \\
& i \hbar \frac{\partial C_{m}^{(2)}(\vec{R}(t), t)}{\partial t}=\left(\sum+\int\right)_{n} W_{m n}^{21}(R(t), t) \gamma_{n m}^{(12) *}(t) C_{n}^{(1)}(\vec{R}(t), t),
\end{aligned}
$$

where

$$
\gamma_{n m}^{(j k)}(t)=e^{i\left(E_{n}^{(j 3)}-E_{m}^{(k 3)}\right) t / \hbar}(j \neq k=1,2) .
$$

The matrix elements $\mathcal{W}_{n m}^{j k}(R(t), t)$ are obtained by integrating the potentials in Eqs. (5)-(6) between the channel functions (16),

$$
W_{n m}^{j k}(R(t), t)=\left\langle e^{i m_{n} \vec{v}_{j} \vec{r} / \hbar-i \frac{m_{n}}{2} v_{j}^{2} t / \hbar} \varphi_{n}^{(j 3)}\left(\vec{r}-\vec{R}_{j}(t)\right)\left|V_{j 3}\left(\vec{r}-\vec{R}_{j}(t)\right)\right| e^{i m_{n} \vec{v}_{k} \vec{r} / \hbar-i \frac{m_{n}}{2} v_{k}^{2} t / \hbar} \varphi_{m}^{(k 3)}\left(\vec{r}-\vec{R}_{k}(t)\right)\right\rangle .
$$


The Eqs. (5)-(6) are then to be solved under the initial conditions

$$
\Psi_{1}(\vec{r}, \vec{R}(t), t) \underset{t \rightarrow-\infty}{\sim} \Phi_{1 s}^{(13)}(\vec{r}, \vec{R}(t), t), \Psi_{2}(\vec{r}, \vec{R}(t), t) \underset{t \rightarrow-\infty}{\sim} 0,
$$

which implies that for the coefficients $C_{n}^{j}(\vec{R}(t), t)$ :

$$
C_{n}^{(1)}(\vec{R}(t), t) \underset{t \rightarrow-\infty}{\sim} \delta_{n 1}, C_{n}^{(2)}(\vec{R}(t), t) \underset{t \rightarrow-\infty}{\sim} 0 .
$$

For reactions at low energies the relative nuclear velocities are practically zero in the respective unities. The exponential factor in Eq. (16), hence, can be replaced by the unit and the matrix elements (21) simplifies to:

$$
\mathcal{W}_{n m}^{j k}(R(t))=\int d^{3} r \varphi_{n}^{(j 3)^{*}}\left(\vec{r}-\vec{R}_{j}(t)\right) V_{j 3}\left(\vec{r}-\vec{R}_{j}(t)\right) \varphi_{m}^{(k 3)}\left(\vec{r}-\vec{R}_{k}(t)\right) .
$$

In order to obtain the capture probabilities $\left|C_{n}^{(2)}(t \sim \infty)\right|^{2}$ we have to solve the system of coupled ordinary differential equations (18)-(19). Note that its ingredients and initial conditions are described in (20), (23) and (24). When solving the resulting coupled set of equations for the expansion coefficients, it is observed that its solutions $C_{n}^{(k)}(\vec{R}(t), t)$ tend toward an asymptotic value $C_{n}^{(k)}(\rho)$ which depends, of course, on the impact parameter $\rho$.

The elastic and transfer semiclassical cross sections of the three-particle collisions are [19]:

$$
\left(\frac{d \sigma}{d \Omega}\right)_{e l}=\left(\frac{d \sigma}{d \Omega}\right)_{c l}\left|C_{1}^{(1)}(\rho)-1\right|^{2},
$$

and

$$
\left(\frac{d \sigma}{d \Omega}\right)_{t r}=\left(\frac{d \sigma}{d \Omega}\right)_{c l}\left|C_{n}^{(2)}(\rho)\right|^{2},
$$

respectively, where $(d \sigma / d \Omega)_{c l}$ is the cross section of the classical scattering of $\mathrm{A}_{1}$ and $\mathrm{A}_{2}$, which are the heavy nuclear cores. For the break-up channel of the reaction one can delineate:

$$
W_{b-u p}\left(\vec{k}_{0}, \rho\right)=\left|\left\langle\vec{k}_{0}(t) \mid \Psi_{1}(\vec{r}, \vec{R}(t), t)+\Psi_{2}(\vec{r}, \vec{R}(t), t)\right\rangle\right|_{t \rightarrow \infty}^{2},
$$

where $W_{b-u p}$ is the neutron emission probability and $\left|\vec{k}_{0}(t)\right\rangle$ is its wave function in the continuous spectrum:

$$
\left|\vec{k}_{0}(t)\right\rangle=\frac{e^{i\left(\vec{k}_{0} \vec{r}-E_{0} t\right) / \hbar}}{\sqrt{(2 \pi)^{3}}},
$$

that is:

$$
\begin{aligned}
W_{b-u p}\left(\vec{k}_{0}, \rho\right) & =\frac{1}{(2 \pi)^{3}} \mid \int d r^{3} e^{-i / \hbar\left(\vec{k}_{0} \vec{r}-E_{0} t\right)}\left(\sum+\iint_{n} C_{n}^{(1)}(\rho) \Phi_{n}^{(13)}(\vec{r}, \vec{R}(t), t)\right. \\
& +\int d r^{3} e^{-i / \hbar\left(\vec{k}_{0} \vec{r}-E_{0} t\right)}\left(\sum+\left.\iint_{m} C_{m}^{(2)}(\rho) \Phi_{m}^{(23)}(\vec{r}, \vec{R}(t), t)\right|^{2} .\right.
\end{aligned}
$$

Finally one can obtain the following formula for the three-body break-up, i.e. neutron emission process:

$$
\begin{aligned}
W_{b-u p}\left(\vec{k}_{0}, \rho\right) & =\frac{1}{(2 \pi)^{3}} \mid\left(\sum+\int\right)_{n} C_{n}^{(1)}(\rho) e^{i E_{0} t / \hbar-i \frac{m_{n}}{2} v_{1}^{2} t / \hbar} \int d r^{3} e^{-i \vec{k}_{0} \vec{r}} e^{i / \hbar m_{n} \vec{v}_{1} \vec{r} / \hbar} \\
& \times \varphi_{n}^{(13)}\left(\vec{r}-\vec{R}_{1}(t)\right)+\left(\sum+\int\right)_{n} C_{n}^{(2)}(\rho) e^{i E_{0} t / \hbar-i \frac{m_{n}}{2} v_{2}^{2} t / \hbar} \\
& \times\left.\int d r^{3} e^{-i \vec{k}_{0} \vec{r}} e^{i / \hbar m_{n} \vec{v}_{2} \vec{r} / \hbar} \varphi_{n}^{(23)}\left(\vec{r}-\vec{R}_{2}(t)\right)\right|^{2}
\end{aligned}
$$


The triple-differential cross section of this process is [19]:

$$
\left(\frac{d^{3} \sigma}{k_{0}^{2} d k_{0} d^{2} \Omega}\right)_{b-u p}=\left(\frac{d \sigma}{d \Omega}\right)_{c l} W_{b-u p}\left(\vec{k}_{0}, \rho\right) .
$$

\subsection{Application of Pechukas's self-consistent approach}

To obtain the true trajectories of the heavy classical particles or nuclear cores $\mathrm{A}_{1}\left(Z_{A_{1}}, M_{A_{1}}\right)$ and $\mathrm{A}_{2}\left(Z_{A_{2}}, M_{A_{2}}\right)$ one can employ the Pechukas self-consistent method [19] based on the Feynman pathintegral theory [20]. In accordance with the method a reduced propagator containing exact information about the reaction $\beta \rightarrow \alpha$ can be written using continual integration

$$
G_{\alpha \beta}\left(\vec{R}_{2} t_{2} \mid \vec{R}_{1} t_{1}\right)=\int_{\vec{R}_{1} t_{1}}^{\vec{R}_{2} t_{2}} D[\vec{R}(t)] e^{i S_{0}[\vec{R}(t)] / \hbar} T_{\alpha \beta}[\vec{R}(t)],
$$

where $S_{0}[\vec{R}(t)]$ is the classical action of the heavy particle moving along $\vec{R}(t) ; T_{\alpha \beta}[\vec{R}(t)]$ are the transition amplitudes used for finding a quantum particle at $t_{2}$ in the state $|\alpha\rangle$ if at $t_{1}$ it was in the state $|\beta\rangle$. Obviously $T_{\alpha \beta}$ is related with the model which was discussed above and determined from Eqs.(18) (23). Now one should impose a proper limit for the scattering or transfer problem, i. e. at $t \rightarrow \infty$. $D[\vec{R}(t)]$ is the measure of continual integration. The time-dependent behaviour of the amplitudes $T_{\alpha \beta}$ is determined by the Hamiltonian $h(t)$ [19]:

$$
h(t)=-\frac{\hbar^{2}}{2 m_{n_{0}}} \triangle_{\vec{r}}+V_{13}\left(\left|\vec{r}-\vec{R}_{1}^{t}\right|\right)+V_{23}\left(\left|\vec{r}-\vec{R}_{2}^{t}\right|\right)+\mathcal{U}\left(R_{12}^{t}\right) .
$$

In accordance with the self-consistent method [19] a basic variational principle is:

$$
\delta\left(S_{0}[\vec{R}(t)]+\hbar \operatorname{Im} \ln T_{\alpha \beta}[\vec{R}(t)]\right)=0 .
$$

Variation of Eq. (34) gives the Newton equations for the dynamics of classical particles in the effective potential field $\mathcal{V}(\vec{R}(t))$. It takes into account an interplay between the classical and quantum degrees of freedom in the semiclassical system, i.e. quantum-mechanical corrections from the third particle $\mathrm{n}$ [19]:

$$
M \frac{d^{2} \vec{R}(t)}{d t^{2}}+\vec{\nabla}_{R} \mathcal{V}(\vec{R}(t))=0
$$

where

$$
\mathcal{V}(\vec{R}(t))=\operatorname{Re} \frac{\left\langle\alpha\left(t, t^{\prime \prime}\right)|h(t)| \beta\left(t, t^{\prime}\right)\right\rangle}{\left\langle\alpha\left(t, t^{\prime \prime}\right) \mid \beta\left(t, t^{\prime}\right)\right\rangle} .
$$

Here, $\left|\alpha\left(t, t^{\prime \prime}\right)\right\rangle$ and $\left|\beta\left(t, t^{\prime}\right)\right\rangle$ are two solutions of the time-dependent Schrödinger equation with different boundary conditions [19]:

$$
i / \hbar \frac{\partial}{\partial t}\left|\alpha\left(t, t^{\prime \prime}\right)\right\rangle=h(t)\left|\alpha\left(t, t^{\prime \prime}\right)\right\rangle,\left|\alpha\left(t^{\prime \prime}, t^{\prime \prime}\right)\right\rangle=|\alpha\rangle,
$$

and

$$
i / \hbar \frac{\partial}{\partial t}\left|\beta\left(t, t^{\prime}\right)\right\rangle=h(t)\left|\beta\left(t, t^{\prime}\right)\right\rangle,\left|\beta\left(t^{\prime}, t^{\prime}\right)\right\rangle=|\beta\rangle .
$$

Therefore

$$
T_{\alpha \beta}\left[\vec{R}_{t}\right]=\left\langle\alpha\left(t, t^{\prime \prime}\right) \mid \beta\left(t, t^{\prime}\right)\right\rangle .
$$


Next, because the Coulomb potential $\mathcal{U}_{c}\left(R_{t}\right)$ between $\mathrm{A}_{1}$ and $\mathrm{A}_{2}$ is a constant (c-number) in the quantum $\vec{r}$-space one can write down:

$$
\mathcal{V}\left(R_{t}\right)=\mathcal{U}_{c}\left(R_{t}\right)+W_{\text {quant }}\left(R_{t}\right)
$$

where

$$
W_{\text {quant }}\left(R_{t}\right)=\operatorname{Re} \frac{\left\langle\alpha\left(t, t^{\prime \prime}\right)|H(t)| \beta\left(t, t^{\prime}\right)\right\rangle}{T_{\alpha \beta}\left[\vec{R}_{t}\right]} .
$$

The three-body hamiltonian is:

$$
H(t)=-\frac{\hbar^{2}}{2 m_{n_{0}}} \Delta_{\vec{r}}+V_{13}\left(\left|\vec{r}-\vec{R}_{1}^{t}\right|\right)+V_{23}\left(\left|\vec{r}-\vec{R}_{2}^{t}\right|\right) .
$$

Thus, the classical part of the three-body problem $\left(\mathrm{A}_{1}, \mathrm{~A}_{2}, \mathrm{n}\right)$ can be resolved in a self-consistent way. In practice, it can be realized, for example, in a few iterations: 1) for an arbitrary $R^{(0)}(t)$, e.g. straightline trajectories, we solve the quantum part of the problem. The Eqs.(18) - (23) should be solved to get the unknown amplitudes $C_{n}^{1}(t \rightarrow \infty)$ and $C_{n}^{2}(t \rightarrow \infty)$ as time-dependent functions: 2) now the effective potential $\mathcal{V}(\vec{R}(t))$ can be computed. To determine $R^{(1)}(t)$ one can employ the expression [30]

$$
t=M \int_{r_{m}}^{R^{\prime}} \frac{R d R}{\sqrt{2 M R^{2}\left(E-\mathcal{V}^{(1)}(R)\right)-J^{2}}},
$$

where $J=\rho \sqrt{2 M E}$; $\rho$ is an impact parameter; $E$ is a collision energy and $M$ is the reduced mass of the nuclear cores $M=M_{A_{1}} M_{A_{2}} /\left(M_{A_{1}}+M_{A_{2}}\right)$. Within the next step one needs to compute the $R_{j}$ points as a function of time $t_{j}$. In order to obtain a smooth function one can make a spline $R^{(1)}(t)=$ $\sum_{\alpha} Z_{\alpha j}\left(t-t_{j}\right)^{\alpha}, t_{j} \leq t \leq t_{j+1}$ and obtain a first approximation for $R^{(1)}(t)$, and so on $(\mathrm{i}=1,2,3, .$. iterations). The cross-section for the reaction is [19], [30]:

$$
\left(\frac{d \sigma}{d \Omega}\right)=\left(\frac{d \sigma}{d \Omega}\right)_{c l}\left|T_{\alpha \beta}[\vec{R}(t)]\right|^{2}
$$

where

$$
\left(\frac{d \sigma}{d \Omega}\right)_{c l}=\frac{\rho(\theta) \csc (\theta)}{|d \theta / d \rho|}
$$

and

$$
\theta(\rho)=\pi-2 \int_{r_{m}}^{\infty} \frac{d R}{R^{2} \sqrt{\rho^{-2}\left(1-\mathcal{V}^{(I)} / E\right)-R^{-2}}},
$$

where $r_{m}$ is a maximum of $R$ when the root is zero.

\section{Quotient analytical solution of the semiclassical Faddeev equations}

In this section we consider a special case of a neutron transfer reaction when the heavy nuclei $\mathrm{A}_{1}\left(Z_{A_{1}}, M_{A_{1}}\right)$ and $\mathrm{A}_{2}\left(Z_{A_{2}}, M_{A_{2}}\right)$ are identical particles, i.e. $\mathrm{A}_{1}\left(Z_{A_{1}}, M_{A_{1}}\right) \equiv \mathrm{A}_{2}\left(Z_{A_{2}}, M_{A_{2}}\right)$, and then we restrict ourselves to the two-level approximation in the expansion (15), i.e. $n=m=1$. To describe the matrix elements (24) we delineate:

$$
\mathcal{W}_{n m}^{12}(R(t), t)=\mathcal{W}_{m n}^{21}(R(t), t)=\mathcal{W}_{11}(R(t), t)=\mathcal{W}(R(t), t),
$$


and the binding energies in the two channels are equal too: $E_{n=1}^{(13)}=E_{m=1}^{(23)}$, therefore, the exponential factors (20), i.e. $\gamma_{n m}^{(12)}(t)$, in the non-stationary Eqs. (18)-(19) are equal to 1.

Below we will demonstrate that the few-body Faddeev equations in the semiclassical model, i.e. Eqs. (18)-(19) can be resolved in an explicit way within the special case mentioned above and the two-level approximation. First of all, in the framework of the model we obtain the following two coupled time-dependent equations:

$$
\begin{aligned}
& \frac{\partial C_{n=1}^{(1)}(\vec{R}(t), t)}{\partial t}=i \mathcal{W}(R(t), t) C_{m=1}^{(2)}(\vec{R}(t), t), \\
& \frac{\partial C_{m=1}^{(2)}(\vec{R}(t), t)}{\partial t}=i \mathcal{W}(R(t), t) C_{n=1}^{(1)}(\vec{R}(t), t) .
\end{aligned}
$$

The two unknown functions $C_{n=1}^{(1)}(\vec{R}(t), t)$ and $C_{m=1}^{(2)}(\vec{R}(t), t)$ can be represented through four new unknown real functions as follows:

$$
C_{n=1}^{(1)}(\vec{R}(t), t)=x_{\vec{R}^{t}}^{(1)}(t)+i y_{\vec{R}^{t}}^{(1)}(t)
$$

and

$$
C_{m=1}^{(2)}(\vec{R}(t), t)=x_{\vec{R}^{t}}^{(2)}(t)+i y_{\vec{R}^{t}}^{(2)}(t) .
$$

Here the $x$ 's and $y$ 's are new unknown real functions. After several algebraic manipulations, one can obtain the following set of four-coupled differential equations:

$$
\begin{aligned}
& \frac{\partial x_{\vec{R}^{t}}^{(1)}(t)}{\partial t}=-W(R(t)) y_{\vec{R}^{t}}^{(2)}(t), \\
& \frac{\partial y_{\vec{R}^{t}}^{(1)}(t)}{\partial t}=W(R(t)) x_{\vec{R}^{t}}^{(2)}(t), \\
& \frac{\partial x_{\vec{R}^{t}}^{(2)}(t)}{\partial t}=-W(R(t)) y_{\vec{R}^{t}}^{(1)}(t), \\
& \frac{\partial y_{\vec{R}^{t}}^{(2)}(t)}{\partial t}=W(R(t)) x_{\vec{R}^{t}}^{(1)}(t) .
\end{aligned}
$$

Now, in accord with the initial conditions (23) one can write down the following four equations for new initial conditions:

$$
x_{\vec{R}^{t}}^{(1)} \underset{t \rightarrow-\infty}{\sim} 1, \quad y_{\vec{R}^{t}}^{(1)}(t) \underset{t \rightarrow-\infty}{\sim} 0
$$

and

$$
x_{\vec{R}^{t}}^{(2)} \underset{t \rightarrow-\infty}{\sim} 0, \quad y_{\vec{R}^{t}}^{(2)}(t) \underset{t \rightarrow-\infty}{\sim} 0 .
$$

Thereby we obtain a new complete set of four-coupled differential equations (52)-(55) with new initial conditions: (56)-(57). Also, one can see now that the four equations are partially uncoupled, i.e. we obtain two pairs of equations. Let us consider the first one: equations (52) and (55). It is obvious, these equations can be written in the following way:

$$
\frac{\partial}{\partial t}\left(x_{\vec{R}^{t}}^{(1)}(t)+i y_{\vec{R}^{t}}^{(2)}(t)\right)=i W(R(t))\left(x_{\vec{R}^{t}}^{(1)}(t)+i y_{\vec{R}^{t}}^{(2)}(t)\right) .
$$


Now one can introduce a new variable

$$
\zeta_{\vec{R}^{t}}(t)=x_{\vec{R}^{t}}^{(1)}(t)+i y_{\vec{R}^{t}}^{(2)}(t)
$$

and solve the equation for $\zeta_{\vec{R}^{t}}(t)$ via separation of variables, i.e.

$$
\frac{\partial \zeta_{\vec{R}^{t}}(t)}{\zeta_{\vec{R}^{t}}(t)}=i W(R(t)) \partial t
$$

A general solution to the equation above is:

$$
\int_{-\infty}^{t} \frac{\partial \zeta_{\vec{R}^{t}}(t)}{\zeta_{\vec{R}^{t}}(t)}=i \int_{-\infty}^{t} W(R(t)) \partial t+C, \quad \text { or } \quad \zeta_{\vec{R}^{t}}(t)=\exp \left\{i \cdot \int_{-\infty}^{t} W(R(t)) \partial t+C\right\} .
$$

Taking into account the initial conditions (56)-(57) and the Eq. (59) one can define $C=0$.

With regard to the second pair of the differential equations, namely (53)-(54), one can carry out the same consideration. However, in this case the initial conditions for the functions $y_{\vec{R}^{t}}^{(1)}(t)$ and $x_{\vec{R}^{t}}^{(2)}(t)$ in (56)-(57) cannot be satisfied, since they are both equal to zero. Therefore these two functions should be excluded from the final solution. Thus, the final solution of the coupled equations (48)-(49) is the following:

$$
\begin{aligned}
& C_{n=1}^{(1)}(\vec{R}(t), t)=C^{\left(A_{1}, n\right)}(\vec{R}(t), t)=\cos \left(\int_{-\infty}^{t} \mathcal{W}\left(R\left(t^{\prime}\right)\right) d t^{\prime}\right), \\
& C_{m=1}^{(2)}(\vec{R}(t), t)=C^{\left(A_{2}, n\right)}(\vec{R}(t), t)=i \sin \left(\int_{-\infty}^{t} \mathcal{W}\left(R\left(t^{\prime}\right)\right) d t^{\prime}\right) .
\end{aligned}
$$

One can see that the important unitarity condition is also satisfied in this consideration, i.e.

$$
\left|C_{n=1}^{(1)}(\vec{R}(t), t)\right|^{2}+\left|C_{m=1}^{(2)}(\vec{R}(t), t)\right|^{2}=1
$$

In Eqs. (62)-(63) we have introduced new notation for the semiclassical amplitudes in the two-level approximation: $C^{\left(A_{1}, n\right)}$ is responsible for the process in which the neutron remains in a bound state with the heavy nucleus $\mathrm{A}_{1}$ after the collision, and $C^{\left(A_{2}, n\right)}$ is responsible for the neutron transfer process from $\mathrm{A}_{1}$ to $\mathrm{A}_{2}$. As one can see these amplitudes have an explicit dependence on time $t$ and also they have a parametrical dependence on the shape of the classical trajectories $\vec{R}(t)$ of the heavy particles.

Now, taking into account that:

$$
d t=\frac{M R d R}{\sqrt{2 M R^{2}\left(E-\mathcal{V}\left(R_{t}\right)\right)-J^{2}}},
$$

where $\mathcal{V}\left(R_{t}\right)=\mathcal{U}_{c}\left(R_{t}\right)+W_{\text {quant }}\left(R_{t}\right), \mathcal{U}_{c}\left(R_{t}\right)=\left(Z_{A_{1}} Z_{A_{2}} e^{2}\right) / R_{t}, E$ is the c.m. collision energy, $M$ is the reduced mass, $e$ is the elementary charge. Thus, the three-body transfer cross section can be written as:

$$
\left(\frac{d \sigma}{d \Omega}\right)_{t r}=\left(\frac{d \sigma}{d \Omega}\right)_{c l} \hbar^{2} \sin ^{2}\left(2 M \int_{r_{m}}^{+\infty} \frac{W(R) R d R}{\sqrt{2 M R^{2}\left(E-\mathcal{V}\left(R_{t}\right)\right)-2 M E \rho^{2}(\theta)}}\right) .
$$

Here $\theta$ is the scattering angle of the classical particles [30]:

$$
\rho(\theta)=\frac{\cot (\theta / 2)}{2 E},
$$


and also from [30]:

$$
\left(\frac{d \sigma}{d \Omega}\right)_{c l}=\left(\frac{Z_{A_{1}} Z_{A_{2}} e^{2}}{4 E \sin ^{2}(\theta / 2)}\right)^{2}
$$

Thereby our final result for the semiclassical three-body neutron transfer cross-section is:

$$
\sigma_{t r}(E)=\int_{0}^{\pi} \sin \theta d \theta \sin ^{2}\left(\int_{r_{m}}^{+\infty} \frac{4 M \pi \hbar^{2} \mathcal{W}(R) R d R}{\sqrt{2 M R^{2}\left(E-\mathcal{V}\left(R_{t}\right)\right)-2 M E \rho^{2}(\theta)}}\right)\left(\frac{Z_{A_{1}} Z_{A_{2}} e^{2}}{4 E \sin ^{2}(\theta / 2)}\right)^{2} .
$$

\subsection{The effective quantum-classical potential between heavy particles $A_{1}$ and $A_{2}$}

Let us now proceed to a calculation of the effective "quantum-classical" potential $\mathcal{V}(R(t))$ between $\mathrm{A}_{1}$ and $\mathrm{A}_{2}$ in the transfer channel. To define amplitudes for the reaction we have to adopt the limit $t \rightarrow \infty$ which is equivalent to $t=t^{\prime \prime}$ in Eq. (39)

$$
T_{\alpha \beta}\left[\vec{R}_{t}\right]=\left\langle\alpha \mid \beta\left(t^{\prime}, t^{\prime \prime}\right)\right\rangle,
$$

here $|\alpha\rangle$ corresponds to the outgoing $\left(\mathrm{A}_{2}, \mathrm{n}\right)$-bound state wave function

$$
|\alpha\rangle=\left.\left|\Phi_{v}^{\left(A_{2}, n\right)}\left(\left|\vec{r}-\vec{R}_{2}(t)\right|\right)\right\rangle\right|_{t \rightarrow \infty}
$$

where $v$ denotes a quantum state of the system $\left(\mathrm{A}_{2}, \mathrm{n}\right)$, e.g. $v=1$. Next, $\left|\beta\left(t^{\prime}, t^{\prime \prime}\right)\right\rangle$ corresponds to the total wave function of our three-particle system:

$$
\left|\beta\left(t^{\prime}, t^{\prime \prime}\right)\right\rangle=\left.|\Psi(t)\rangle\right|_{t \rightarrow \infty} \approx\left[C^{\left(A_{1}, n\right)}(t)\left|\Phi_{v}^{\left(A_{1}, n\right)}\left(\left|\vec{r}-\vec{R}_{1}(t)\right|\right)\right\rangle+C^{\left(A_{2}, n\right)}(t)\left|\Phi_{v}^{\left(A_{2}, n\right)}\left(\left|\vec{r}-\vec{R}_{2}(t)\right|\right)\right\rangle\right]_{t \rightarrow \infty},
$$

and for the nucleon transfer channel we have:

$$
T_{\alpha \beta}[\vec{R}(t)]=C^{\left(A_{2}, n\right)}(\infty)
$$

Thus, the effective potential is:

$$
\begin{aligned}
\mathcal{V}\left(R_{t}\right) & =\left(Z_{A_{1}} Z_{A_{2}}\right) e^{2} / R+\operatorname{Re}\left[C^{\left(A_{1}, n\right)}(\infty) / C^{\left(A_{2}, n\right)}(\infty)\left\{\left\langle\Phi_{v}^{\left(A_{2}, n\right)}(\vec{y})\left|p^{2} / 2 m_{n}+V_{13}(x)\right| \Phi_{v}^{\left(A_{1}, n\right)}(\vec{x})\right\rangle\right.\right. \\
& \left.+\left\langle\Phi_{v}^{\left(A_{2}, n\right)}(\vec{y})\left|V_{23}(y)\right| \Phi_{v}^{\left(A_{1}, n\right)}(\vec{x})\right\rangle\right\}+\left\{\left\langle\Phi_{v}^{\left(A_{2}, n\right)}(\vec{y})\left|p^{2} / 2 m_{n}+V_{23}(y)\right| \Phi_{v}^{\left(A_{2}, n\right)}(\vec{y})\right\rangle\right. \\
& \left.\left.+\left\langle\Phi_{v}^{\left(A_{2}, n\right)}(\vec{y})\left|V_{13}(x)\right| \Phi_{v}^{\left(A_{2}, n\right)}(\vec{y})\right\rangle\right\}\right]
\end{aligned}
$$

with $\vec{y}=\vec{r}-\vec{R}_{2}(t)$ and $\vec{x}=\vec{r}-\vec{R}_{1}(t)$. Finally one can obtain:

$$
\begin{aligned}
\mathcal{V}\left(R_{t}\right) & =\left(Z_{A_{1}} Z_{A_{2}}\right) e^{2} / R+\operatorname{Re}\left[C^{\left(A_{1}, n\right)}(\infty) / C^{\left(A_{2}, n\right)}(\infty) \int d^{3} \vec{r}\left(\Phi_{v}^{\left(A_{2}, n\right)}(\vec{y})\right)^{*} V_{23}(y)\right. \\
& \left.\times \Phi_{v}^{\left(A_{1}, n\right)}(\vec{x})+\int d^{3} \vec{r}\left(\Phi_{v}^{\left(A_{2}, n\right)}(\vec{y})\right)^{*} V_{13}(x) \Phi_{v}^{\left(A_{2}, n\right)}(\vec{y})\right]
\end{aligned}
$$

where amplitudes $C^{\left(A_{1}, n\right)}$ and $C^{\left(A_{2}, n\right)}$ are from Eqs. (62)-(63).

Now one can do the following: in the first step (we could call it a zero-th (0-th) approximation) we only retain the Coulomb interaction in the effective potential $\mathcal{V}_{(0)}\left(R_{t}\right)=\left(Z_{A_{1}} Z_{A_{2}}\right) e^{2} / R$, and then calculate the 0 -th order approximation to the amplitudes $C_{(0)}^{\left(A_{1}, n\right)}(\infty)$ and $C_{(0)}^{\left(A_{2}, n\right)}(\infty)$. In the second step we can then compute the quantum corrections to the effective potential $V_{(1)}\left(R_{t}\right)$. Then in the first approximation one can compute the amplitudes $C_{(1)}^{\left(A_{1}, n\right)}(\infty)$ and $C_{(1)}^{\left(A_{2}, n\right)}(\infty)$ and, of course, one could continue the process if desired. 
Let us delineate the matrix element $\mathcal{W}(R(t))$, i.e.

$$
\mathcal{W}(R(t))=\int d^{3} r\left(\varphi_{v}^{\left(A_{j}, n\right)}\left(\vec{r}-\vec{R}_{j}(t)\right)\right)^{*} V_{j 3}\left(\vec{r}-\vec{R}_{j}(t)\right) \varphi_{v}^{\left(A_{k}, n\right)}\left(\vec{r}-\vec{R}_{k}(t)\right),
$$

where $V_{j 3}(x)$ is a local interaction, e.g. a potential well for the bound system ${ }^{16} \mathrm{O}-\mathrm{n}$ which gives bound states and $\varphi_{v}^{\left(A_{j}, n\right)}\left(\vec{r}-\vec{R}_{j}(t)\right)$ are its wave functions. The results obtained can be used to describe the one n-transfer reaction between two ${ }^{16} \mathrm{O}$ nuclei. This example has also been numerically calculated in $[11,12]$ using single-term separable potentials and straight-line trajectories. In the framework of the current formalism the simple expressions (66)-(69) and (76) have been derived by taking into account the Coulomb potential between nuclear cores and using the local nuclear potentials between $\mathrm{A}_{i}$ and $\mathrm{n}(i=1,2)$. The elastic and transfer reaction cross-sections are obtained using the self-consistent Pechukas method. In turn the Pechukas method takes into account the interplay between the classical and quantum degrees of freedom in the semiclassical system and is consistent with the conservation laws of energy and angular momentum [19].

It is essential to note here, that the same considerations as above could be carried out for the threebody break-up channel. This is a very attractive and complicated problem in the field of heavy-ion collisions, namely, for a neutron emission reaction and/or a charged particle, such as the $\alpha$-particle emission process. In the case of such reactions, for instance the $\alpha$-particle emission, in Eqs. (27)-(30) we would need to apply Coulomb asymptotic wave function in the three-body continuum. Obviously, the effective potential between the heavy particles $\mathrm{A}_{1}$ and $\mathrm{A}_{2}$ will also be different in the three-body break-up channel.

\section{Conclusion}

We have formulated a semiclassical approach for a model three-body system with two heavy nuclear cores $A_{1}$ and $A_{2}$ moving along classical trajectories and a lighter particle n, i.e. a neutron. The three-body system is shown in Fig. 1. The quantum dynamics of the neutron is described based on the few-body quantum-mechanical Eqs. (5)-(6) with realistic (local) nuclear-nuclear potentials $V_{13}(\vec{x})$ and $V_{23}(\vec{y})$. The classical dynamics of $\mathrm{A}_{1}$ and $\mathrm{A}_{2}$ are described based on Newtonian (non-relativistic) mechanics Eq. (35). However, this becomes important with the use of the Pechukas self-consistent method, where we could take into account the interplay between classical and quantum degrees of freedom in the system and thereby obtain even more realistic trajectories for the classical particles. Therefore, the proposed method is divided into two parts: the 1st part is the quantum-mechanical problem for a lighter particle " $\mathrm{n}$ " dipped into the nuclear potential wells of the heavy particles $\mathrm{A}_{1}$ and $\mathrm{A}_{2}$, the second part is the classical problem for two heavy nuclear cores interacting by the quantum and classical (Coulomb) self-consistent potential $\mathcal{V}(\vec{R}(t)$ ), i.e. Eqs. (40)-(41). Also, it would be appropriate to make a few comments about the semiclassical Faddeev-type equations, i.e. Eqs. (5)(6). First of all, the constructed coupled equations satisfy the Schrödinger equation exactly. Secondly, the Faddeev decomposition avoids the over-completeness problem. Therefore, two-body subsystems are treated in an equivalent way and the correct asymptotic behavior is guaranteed [24, 25]. The current method simplifies the solution procedure and provides the correct asymptotic behavior of the solution. Finally, the Faddeev-type equations have the same advantages as the original Faddeev equations, because they are formulated for the three-body wave function components $\Psi_{1}(\vec{r}, \vec{R}(t), t)$ and $\Psi_{2}(\vec{r}, \vec{R}(t), t)$ with correct physical asymptotes.

In the solution of the time-dependent Eqs. (5)-(6) one needs to consider the number of channels $n$ which are needed to be included in the close-coupling expansion (15). This is an important issue, because $n$ controls the number of coupled differential equations to be numerically solved, i.e. Eqs. 
(18)-(19). However, in the actual numerical computation one could only retain a few states in Eq. (15). For example, it is quite reasonable to expect that for closed shell nuclei, e.g. $\mathrm{A}_{i} \equiv{ }^{4} \mathrm{He},{ }^{12} \mathrm{C}$ or ${ }^{16} \mathrm{O}$, just one or two states should be predominate during low energy collisions. Next, the expression (16) is true for an inertial coordinate system, i.e. when $v=\dot{\vec{R}}(t)=$ const. In the case of the realistic trajectories $\dot{\vec{R}}_{i}(t) \neq$ const, one needs to make considerable alterations in the expression and in the theory. However, at low energies when $v \approx 0$ the exponent multiplier is approximately equal to one, i.e. $e^{i m_{n} \vec{v} r-i \frac{m_{n}}{2} v^{2} t} \approx 1$.

In conclusion, as mentioned in the introduction, few-body semiclassical models in nuclear physics can help us gain deeper insight into complex nuclear processes. Specifically, in the case of identical heavy nuclei and a two-level approximation in the expansion (15) the resulting set of coupled differential Eqs. (18)-(19) can be resolved analytically, i.e. expressions (62)-(63). This analytical solution might be useful, for example, in the investigation of the nucleus ${ }^{13} \mathrm{C}$, e.g. in the low energy collision:

$$
{ }^{13} \mathrm{C}+{ }^{12} \mathrm{C} \rightarrow{ }^{12} \mathrm{C}+{ }^{13} \mathrm{C}(\mathrm{n}-\text { transfer }) ;{ }^{12} \mathrm{C}+\mathrm{n}+{ }^{12} \mathrm{C}(\mathrm{n}-\text { emission channel }) .
$$

The structure of ${ }^{13} \mathrm{C}=\left({ }^{12} \mathrm{C}, n\right)$ can be important for low energy reactions in the s-process neutron source in stars ${ }^{13} \mathrm{C}(\alpha, n){ }^{16} \mathrm{O}$, see for example [31-33]. Also, we would like to note, that a possible relativistic expansion of the semiclassical theory presented above in this paper would be a very useful future project.

\section{Acknowledgements}

This work was partially supported by the Office of Sponsored Programs and Integrated Science and Engineering Laboratory Facility (ISELF, http://stcloudstate.edu/iself/) of St. Cloud State University, St. Cloud, Minnesota 56301-4498, USA.

\section{References}

[1] J.S. Al-Khalili and J.A. Tostevin, "Few-Body Models of Nuclear Reactions", Chapter 3.1.3, p.1373 in "Scattering: Scattering and Inverse Scattering in Pure and Applied Science". Eds. R. Pike and P. Sabatier, Academic Press, 2002.

[2] W. Leidemann and G. Orlandini, Prog. in Partic. and Nucl. Phys. 68, 158 (2013).

[3] E. Hiyama, S. Ohnishi, M. Kamimura, Y. Yamamoto, Nucl. Phys. A 908, 29 (2013); E. Hiyama, M. Kamimura, Y. Yamamoto, T. Motoba, Phys. Rev. Lett. 104, 212502 (2010).

[4] E. Hiyama, EPJ Web of Conferences 20, 04006 (2012).

[5] A. Deltuva, J. Phys.: Conf. Ser. 205012017 (2010)

[6] D. V. Fedorov and A. S. Jensen, Phys. Lett. B389, 631 (1996).

[7] N.B. Nguyen, F.M. Nunes, and I.J. Thompson, Phys. Rev. C87, 054615 (2013).

[8] S. Ishikawa, Phys. Rev. C87, 055804 (2013).

[9] E. Nielsen, D.V. Fedorov, A.S. Jensen, and E. Garrido, Phys. Rep. 347, 373 (2001).

[10] L. D. Faddeev, Sov. Phys. JETP 12, 1014 (1961).

[11] B. Milek, R. Reif, and J. Revai, Phys. Lett. B150, 65 (1985).

[12] J. Revai, Nucl. Phys. A435, 512 (1985).

[13] B.-A. Li, L.-W. Chen, and C.M. Ko, Phys. Rep. 464113 (2008).

[14] R.A. Malfliet, Classical and Quantum-Mechanical Aspects of Heavy Ion Collisions, SpringerVerlag, 1974, p.86.

[15] J-I. Fujita and T. Negishi, Prog. Theor. Phys. 55, 734 (1976). 
[16] W.R. Phillips, Rep. Prog. Phys. 40, 345 (1977).

[17] D.M. Brink, Phys. Lett. B 40, 37 (1972).

[18] W. von Oertzen, Deep-Inelastic and Fusion Reactions with Heavy Ions, Lect. Notes Phys. 117 (1980).

[19] P. Pechukas, Phys. Rev. 181, 166 (1969); 181, 174 (1969)

[20] R. P. Feynman and A. R. Hibbs, Quantum Mechanics and Path Integrals (McGraw-Hill, New York, 1965)

[21] G. D. Billing, J. Chem. Phys. 86, 2617 (1987).

[22] R. Kapral and G. Ciccotti, J. Chem. Phys. 110, 8919 (1999).

[23] R.A. Sultanov and D. Guster, In Proc.: Computational Science and its Applications - ICCSA 2003, PT 1, Lect. Notes in Comp. Sci. vol. 2667, Springer-Verlag, p.p. 568-576.

[24] R.A. Sultanov and D. Guster, Few-Body Systems 54, 1157 (2013).

[25] R.A. Sultanov and D. Guster, J. Phys. B: At. Mol. Opt. Phys. 46, 215204 (2013).

[26] R. A. Sultanov, Phys. Rev. A 50, 2376 (1994).

[27] R.A. Sultanov, W. Sandhas, and V.B. Belyaev, JINR Rapid Communications (Dubna, Russia), No.5[85]-97, p.p. 91-98 (1997).

[28] R. A. Sultanov, W. Sandhas, and V. B. Belyaev, Euro. Phys. J. D 5, 33 (1999).

[29] L. D. Landau and E. M. Lifshitz, Course of Theoretical Physics, Mechanics, Pergamon Press, 1960.

[30] R. G. Newton, Scattering Theory of Waves and Particles, McGraw-Hill, New York, 1965.

[31] D. Hollowell and I. Iben, Jr, Astrophys. J. 340, 966 (1989); H. W. Drotleff et al., Astrophys. J. 414, 735 (1993).

[32] C. R. Brune, I. Licot and R. W. Kavanagh, Phys. Rev. C48, 3119 (1993).

[33] M. La Cognata et al., Phys. Rev. Lett. 109, 232701 (2012). 